\title{
Clinical Characteristics of Primary Epiploic Appendagitis
}

\author{
Young Un Choi, Pyong Wha Choi, Yong Hwan Park, Jae Il Kim, Tae Gil Heo, Je Hoon Park, \\ Myung Soo Lee, Chul Nam Kim, Surk Hyo Chang, Jeong Wook Seo ${ }^{1}$ \\ Departments of Surgery and ${ }^{1}$ Radiology, Ilsan Paik Hospital, Inje University College of Medicine, Goyang, Korea
}

Purpose: Primary epiploic appendagitis (PEA) is a rare cause of an acute abdomen. It can be clinically misdiagnosed as either diverticulitis or appendicitis on clinical examination because the clinical symptoms and signs of PEA are non-specific. The present study was performed to describe the clinical characteristics of PEA and to assess the differences between PEA and diverticulitis.

Methods: We reviewed the clinical records and radiologic findings of 31 consecutive patients with PEA and compared them with those of patients with diverticulitis without complications.

Results: In most cases, abdominal pain was localized to the right (13 cases, $41.9 \%)$ or left (13 cases, $41.9 \%)$ lower quadrants. Gastrointestinal symptoms such as nausea and vomiting were infrequent, and localized tenderness without peritoneal irritation was common. All patients were afebrile, and only 4 patients (12.9\%) showed leukocytosis. In all cases except one, a pericolic fatty mass with a hyperattenuated ring was observed on computed tomography. Patients with left PEA were younger than those with diverticulitis ( $41.4 \pm 11.9$ vs. $69.7 \pm 13.3, \mathrm{P}<0.001)$, and the mean body mass index was higher in patients with left PEA ( $26.4 \pm 2.9$ vs. $22.6 \pm 3.4, \mathrm{P}=0.01)$. Whereas one patient $(6.7 \%)$ with left PEA showed leukocytosis, the incidence of leukocytosis in patients with diverticulitis was $80 \%(8 / 10)(\mathrm{P}<0.001)$.

Conclusion: In patients with an acute abdomen showing localized tenderness without associated symptoms or leukocytosis, a high index of suspicion for PEA is necessary. For correct diagnosis and proper management, it would useful for surgeons to be aware of the computed tomographic findings and the natural course of the disease.

Keywords: Epiploic appendagitis; Diverticulitis; Acute abdomen

\section{INTRODUCTION}

Epiploic appendages are fatty appendages originating in two rows parallel to the taenia coli. Primary epiploic appendagitis (PEA) refers to inflammation in the epiploic appendages caused by spontaneous torsion, a hemorrhagic infarct, etc. [1-4]. In general, PEA is a self-limiting disease, and most patients recover with conservative management in less than 10 days. Thus, antibiotics or surgical treatments are rarely required. Nonetheless, because the incidence is low and the clinical characteristics are non-specific, in the past,

Received: March 3, 2011 Accepted: May 31, 2011

Correspondence to: Pyong Wha Choi, M.D.

Department of Surgery, Ilsan Paik Hospital, Inje University College of

Medicine, 2240 Daehwa-dong, Ilsanseo-gu, Goyang 411-706, Korea

Tel: +82-31-910-7622 Fax: +82-31-910-7319

E-mail: peacechoi@paik.ac.kr

(c) 2011 The Korean Society of Coloproctology

This is an open-access article distributed under the terms of the Creative Commons Attribution NonCommercial License (http://creativecommons.org/licenses/by-nc/3.0) which permits unrestricted noncommercial use, distribution, and reproduction in any medium, provided the original work is properly cited. it was misdiagnosed as acute appendicitis or diverticulitis, and a laparotomy was performed in some cases [5-8]. In patients with an acute abdomen, appendicitis and diverticulitis could be considered to be the most common diseases that cause pain in the lower abdomen, and most of such diseases have been diagnosed clinically. With the advancements of radiological techniques, intra-abdominal inflammatory diseases can be diagnosed more accurately. This has led to more timely surgical treatment when it is indicated and to the avoidance of unnecessary surgery for diseases that do not require a laparotomy. In addition, as the characteristic radiological findings of PEA have been reported, its diagnosis without a laparotomy is now possible. However, because its findings on physical examination are similar to those of other intra-abdominal inflammatory diseases, prior to imaging studies, it is putatively diagnosed as acute appendicitis or diverticulitis according to the location of pain in most cases. In addition, as many surgeons are unfamiliar with the disease, it may not be diagnosed even after imaging studies in some cases [9-11]. Thus, we conducted this study to establish guidelines for the diagnosis of PEA by analyzing the characteristics of PEA and by comparing the differences from di- 
verticulitis, which shows similar clinical features depending on the location of pain.

\section{METHODS}

This study was performed on 31 patients who were diagnosed as having PEA at Inje University College of Medicine, Ilsan Paik Hospital, from January 2001 to October 2010. The patients' medical records were retrospectively reviewed and analyzed with regard to the demographic data, the characteristics of abdominal pain, the findings on physical examination, the laboratory results, and the treatment methods. The degree of obesity of the patients was calculated by applying the body mass index. A body temperature at the time of admission higher than $38.0^{\circ} \mathrm{C}$ and a WBC count higher than $10,000 / \mathrm{mm}^{3}$ were defined as fever and leukocytosis, respectively. The size of epiploic appendagitis was defined as the largest diameter on the radiological findings. The location of epiploic appendagitis was divided at the splenic flexure. The right colon was defined as the large bowel, including the cecum, ascending colon, and transverse colon, and the left colon was comprised of portions of splenic flexure and the descending and sigmoid colon. According to the location, right colonic and left colonic PEA were diagnosed in 16 (51.6\%) and 15 (48.4\%) patients, respectively.

During the period of our study, 205 patients diagnosed as having diverticulitis. Among them, excluding 23 patients who underwent emergency surgery due to perforation, 182 patients were managed only by using conservative treatments such as antibiotic treatments. When the patients were divided according to the location in the colon, right colonic diverticulitis was noted in 172 patients (94.5\%), and left colonic diverticulitis was noted in 10 patients (5.5\%). According to location, the clinical characteristics of left and right diverticulitis were compared with those of PEA.

In regard to statistical analysis, correlations of the variables were analyzed by using the chi-square test and the Fisher's exact test. The averages were compared by using the t-test, and P-value less than 0.05 was considered to be significant.

\section{RESULTS}

\section{Clinical characteristics of patients with primary epiploic appendagitis}

The ratio of males to females of patients who were diagnosed as having PEA was 2.4:1, and their mean age was 40 years (range, 20 to 63 years). Eight patients (25.8\%) had underlying diseases such as hypertension and diabetes (Table 1).

The major symptom of all patients was abdominal pain, and none of them was diagnosed incidentally. The mean delay between the onset of symptoms and hospital visit was $2.3 \pm 1.6$ days ( 4 hours- 7 days). The right lower quadrant, the left lower quadrant, and the right upper quadrant of the abdomen and the lower abdomen were the locations of pain in 13 patients (41.9\%), 13 patients (41.9\%), 3 patients (9.7\%) and 2 patients (6.5\%), respectively. There were no aggravating factors for the pain in most cases. However, the pain was aggravated when gas was released in one patient $(3.2 \%)$ and when the body was bent in another patient (3.2\%). None of the patients had a fever, and two patients (6.5\%) had chills. Other symptoms, including anorexia, nausea, vomiting, diarrhea, and constipation were noted in three cases $(9.7 \%)$, four cases $(12.9 \%)$, one case $(3.2 \%)$, two cases $(6.5 \%)$, and four cases $(12.9 \%)$, respectively. Physical examination revealed a localized abdominal tenderness in all patients, and eight patients (25.8\%) showed definite rebound tenderness, but none of the patients showed abdominal rigidity. Leukocytosis was found in four patients (12.9\%) on the laboratory test (Table 1).

\section{Diagnosis of primary epiploic appendagitis and radiological findings}

During the study period, 3 patients (9.7\%) were diagnosed before the year 2006, and 28 patients (90.3\%) were diagnosed thereafter. The presumptive diagnosis made after history taking, physical ex-

Table 1. Clinical features of patients with primary epiploic appendagitis

\begin{tabular}{lc}
\hline Clinical features & No. (\%) \\
\hline Mean age (range, yr) & $40(20-63)$ \\
Sex ratio (male:female) & $2.4: 1(22: 9)$ \\
Underlying disease &
\end{tabular}
$(+)$
$8(25.8)$

$(-)$

$23(74.2)$

Mean body mass index $\left(\mathrm{kg} / \mathrm{m}^{2}\right)$

$25.9 \pm 3.5$

Location of pain

$\begin{array}{lc}\text { Right lower quadrant } & 13(41.9) \\ \text { Left lower quadrant } & 13(41.9) \\ \text { Right upper quadrant } & 3(9.7) \\ \text { Low abdomen } & 2(6.5)\end{array}$

Tenderness

$31(100)$

$(-)$

$0(0)$

Rebound tenderness

$\begin{array}{rr}(+) & 8(25.8) \\ (-) & 23(74.2)\end{array}$

Muscle rigidity

$(+)$

$0(0)$

$(-)$

31 (100)

Fever

\begin{tabular}{lc}
$(+)$ & $0(0)$ \\
$(-)$ & $31(100)$ \\
Leukocytosis & \\
$(+)$ & $4(12.9)$ \\
$(-)$ & $27(87.1)$ \\
\hline
\end{tabular}


amination and laboratory test, but prior to imaging studies, was diverticulitis or appendicitis in most cases; the presumptive diagnosis of three patients (9.7\%) who presented with right upper quadrant pain was cholecystitis, and that of two patients (6.5\%) who presented with lower abdominal pain was pelvic inflammatory disease. Acute mesenteric lymphadenitis, acute gastroenteritis, and a ureter stone were presumptive diagnoses in 1 patient (3.2\%) each (Table 2).

Abdominal ultrasonography (US) was performed in four patients, and it showed an oval noncompressible hyperechoic mass adjacent to the colon directly under the site of tenderness (Fig. 1). In three patients (9.7\%), computed tomography (CT) was performed after abdominal US to confirm the diagnosis. In all patients who underwent CT, an oval fatty mass with a hyperattenuating rim adjacent

Table 2. Presumptive diagnosis prior to radiologic studies

\begin{tabular}{lc}
\hline Impression & No. (\%) \\
\hline Diverticulitis & $13(41.9)$ \\
Appendicitis & $6(19.4)$ \\
Diverticulitis or appendicitis & $4(12.9)$ \\
Cholecystitis & $3(9.7)$ \\
Pelvic inflammatory disease & $2(6.5)$ \\
Mesenteric lymphadenitis & $1(3.2)$ \\
Acute gastroenteritis & $1(3.2)$ \\
Ureter stone & $1(3.2)$ \\
\hline
\end{tabular}

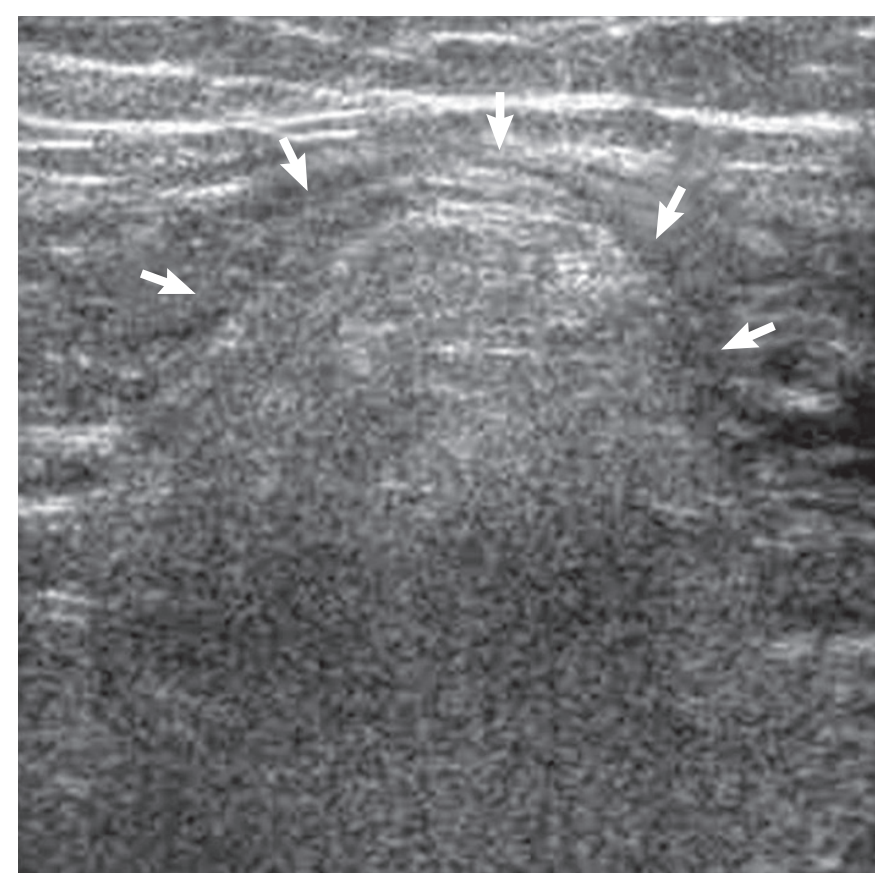

Fig. 1. Ultrasonogram of the left lower quadrant shows a well-defined hyperechoic ovoid mass (arrows) adjacent to the descending colon. to the colon was characteristically detected, and findings of appendicitis, diverticulitis or lymphadenitis were not detected (Fig. 2). Among them, a fine line of high attenuation in the oval fatty mass was found in two patients (6.5\%) (Fig. 3), and an oval fatty mass with a lobulated appearance was observed in one patient (3.2\%).

The ascending colon was the location of PEA in 11 patients $(35.5$ $\%)$, the descending colon in 10 patients (32.3\%), the sigmoid colon in 5 patients $(16.1 \%)$, the cecum in 3 patients $(9.7 \%)$, and the transverse colon in 2 patients (6.5\%). The mean size of PEA was $2.3 \mathrm{~cm}$ (range, 1.0 to $5.5 \mathrm{~cm}$ ).

\section{Treatment and outcomes of primary epiploic appendagitis}

All patients were hospitalized and treated with antibiotics except 1 patient, who was not hospitalized and was managed by using only oral analgesics. The mean duration of antibiotic treatment was 4.3 \pm 1.8 days. Nonsteroidal anti-inflammatory drugs (NSAID) were used in 8 patients (25.8\%) for a mean duration of $2.8 \pm 1.7$ days. Surgery was performed on one patient. The patient presented at another hospital with the chief complaint of left lower quadrant abdominal pain, and based on the CT findings, the presumptive diagnosis was a gastrointestinal stromal tumor (GIST) in the small bowel. The patient was transferred to our hospital, and surgery was performed. However, on laparotomy, the patient was diagnosed as having PEA in the descending colon.

In the patients who could be evaluated from the onset of abdominal pain to the resolution, the mean duration of pain was 4.6 \pm 2.3 days, and the mean hospitalization period was $5.2 \pm 1.9$ days.

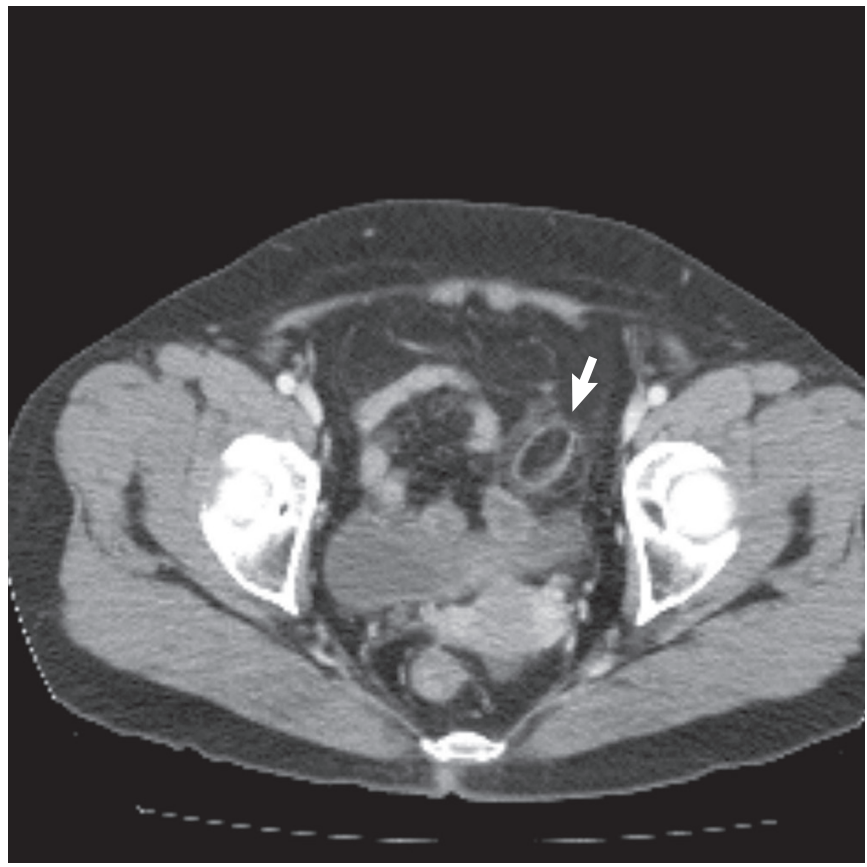

Fig. 2. Computed tomography scan of the low abdomen shows an ovoid fat attenuated mass with hyperattenuated ring (arrow) adjacent to the sigmoid colon. 
Twenty-four patients (77.4\%) were followed up at the outpatient clinic after discharge. The mean follow-up period was $4 \pm 4.7$ weeks.



Fig. 3. Coronary view of the abdominal computed tomography scan shows an ovoid fatty mass with hyperattenuated ring. There is a central high attenuated line (arrow) within the inflamed epiploic appendix. This lesion is believed to be due to thrombosis of the vessel.

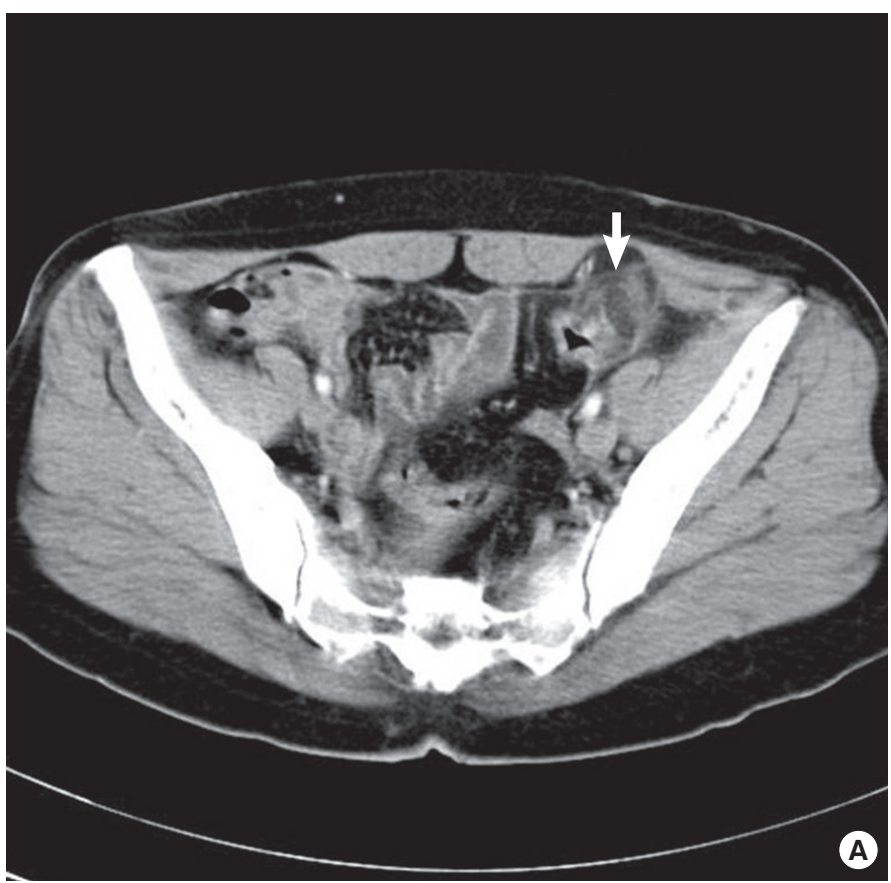

Recurrence of symptoms was not detected in any of patients during the follow-up period. CT was performed in two patients, one at three weeks and the other at four weeks after discharge, and all showed improvement in the inflammation of the epiploic appendage (Fig. 4).

\section{Comparison with acute diverticulitis according to the location}

The patients with left epiploic appendagitis were younger than the patients with left diverticulitis $(41.4 \pm 11.9$ vs. $69.7 \pm 13.3, \mathrm{P}<0.001)$. Gender and underlying disease were not significantly different. The BMI in patients with left epiploic appendagitis was significantly higher $(26.4 \pm 2.9$ vs. $22.6 \pm 3.4, \mathrm{P}=0.01)$ than it was in patients with left diverticulitis. Associated symptoms (anorexia, nausea, vomiting and other gastrointestinal symptoms) were not significantly different between the two groups. None of the patients with epiploic appendagitis had a fever whereas 4 patients with left diverticulitis $(40.0 \%)$ had fever, and this difference was statistically significant $(P=0.017)$. Significant differences were not observed in the findings on physical examination. However, leukocytosis was observed in 8 patients with left diverticulitis, which was significantly different from that in patients with left epiploic appendagitis $(80 \%$ vs. $6.7 \%, \mathrm{P}<0.001)$ (Table 3 ).

When patients with right epiploic appendagitis were compared with patients with right diverticulitis, no differences in demographic factors were observed. The BMI in patients with right epiploic appendagitis was significantly higher than that in patient with right

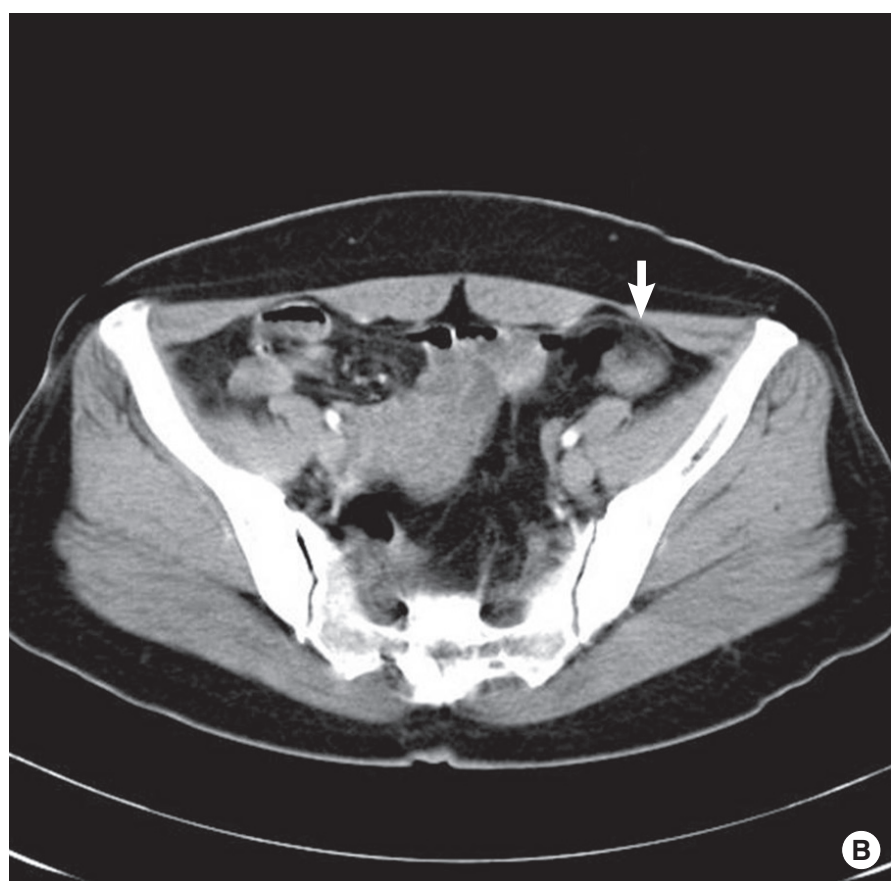

Fig. 4. Follow-up findings of the computed tomography scan of primary epiploic appendagitis. (A) The arrow indicates the inflamed epiploic appendix of the descending colon at admission. (B) The inflamed epiploic appendix of the descending colon is hardly detected in the descending colon four weeks after discharge. 
Table 3. Clinical features of patients with left primary epiploic appendagitis (PEA) and left diverticulitis

\begin{tabular}{lccc}
\hline Variables & $\begin{array}{c}\text { Left PEA } \\
(\mathrm{n}=15)\end{array}$ & $\begin{array}{c}\text { Left diverticulitis } \\
(\mathrm{n}=10)\end{array}$ & P-value \\
\hline Age $(\mathrm{yr})$ & $41.4 \pm 11.9$ & $69.7 \pm 13.3$ & $<0.001$ \\
Sex (male:female) & $10: 5$ & $4: 6$ & 0.241 \\
Underlying disease & $5(33.3)$ & $7(70.0)$ & 0.111 \\
Body mass index & $26.4 \pm 2.9$ & $22.6 \pm 3.4$ & 0.01 \\
Anorexia & $2(13.3)$ & $1(10.0)$ & 1.00 \\
Nausea & $3(20.0)$ & $2(20.0)$ & 1.00 \\
Vomiting & $1(6.7)$ & $2(20.0)$ & 0.543 \\
Diarrhea & $1(6.7)$ & $2(20.0)$ & 0.543 \\
Constipation & $3(20.0)$ & $2(20.0)$ & 1.00 \\
Fever & $0(0.0)$ & $4(40.0)$ & 0.017 \\
Chill & $1(6.7)$ & $1(10.0)$ & 1.00 \\
Tenderness & $15(100.0)$ & $10(100.0)$ & Not available \\
Rebound tenderness & $5(33.3)$ & $4(40.0)$ & 1.00 \\
Muscle rigidity & $0(0.0)$ & $3(30.0)$ & 0.52 \\
Leukocytosis & $1(6.7)$ & $8(80.0)$ & $<0.001$ \\
\hline
\end{tabular}

Values are presented as mean \pm SD or number (\%).

diverticulitis ( $25.6 \pm 4.1$ vs. $22.9 \pm 3.2, \mathrm{P}=0.025)$. Associated symptoms were not different between the two groups. Rebound tenderness was more frequently observed in patient with right diverticulitis than in patients with right epiploic appandatitis. However, this difference was not statistically significant $(36.0 \%$ vs. $18.8 \%, \mathrm{P}=$ $0.164)$. Leukocytosis was found in more than half of the patient with right diverticulits $(99 / 172,57.6 \%)$ whereas 3 patients (18.8\%) with right epiploic appendagitis showed leukocytosis; this difference was statistically significant $(\mathrm{P}=0.003)$ (Table 4$)$.

\section{DISCUSSION}

The epiploic appendages are peritoneal pouches that arise from the serosal surface of the colon originating next to the anterior and the posterior taenia coli. Usually their sizes are $1-2 \mathrm{~cm}$ in thickness and $0.5-5 \mathrm{~cm}$ in length. Approximately 50-100 epiploic appendages are distributed from the cecum to the rectosigmoid junction. They have been reported to play the role of cushions that support the colon and to be involved in immune functions, like a small omentum, and in colonic absorption [12,13]. Epiploic appendagitis is a condition in which the epiploic appendage is inflamed, and the inflammation can be primary or secondary. Secondary epiploic appendagitis is caused by adjacent inflammatory diseases such as diverticulitis, appendicitis, cholecystitis, etc. On the other hand, PEA is a fairly rare condition caused by spontaneous torsion or venous thrombosis of an epiploic appendage, followed by ischemic or hemorrhagic infarction and inflammation [3, 4]. An epiploic appendage receives its blood supply from one or two small end-
Table 4. Clinical features of patients with right primary epiploic appendagitis (PEA) and right diverticulitis

\begin{tabular}{lccc}
\hline Variables & $\begin{array}{c}\text { Right PEA } \\
(\mathrm{n}=16)\end{array}$ & $\begin{array}{c}\text { Right diverticulitis } \\
(\mathrm{n}=172)\end{array}$ & P-value \\
\hline Age (yr) & $39.0 \pm 8.19$ & $37.8 \pm 11.8$ & 0.598 \\
Sex (male:female) & $12: 4$ & $105: 67$ & 0.271 \\
Underlying disease & $3(18.8)$ & $41(23.8)$ & 0.767 \\
Body mass index & $25.6 \pm 4.1$ & $22.9 \pm 3.2$ & 0.025 \\
Anorexia & $1(6.3)$ & $20(11.6)$ & 1.00 \\
Nausea & $1(6.3)$ & $33(19.2)$ & 0.312 \\
Vomiting & $0(0.0)$ & $7(4.1)$ & 1.00 \\
Diarrhea & $1(6.3)$ & $24(14.0)$ & 0.700 \\
Constipation & $1(6.3)$ & $21(12.2)$ & 0.699 \\
Fever & $0(0.0)$ & $9(5.2)$ & 1.00 \\
Chill & $1(6.3)$ & $17(9.9)$ & 1.00 \\
Tenderness & $16(100.0)$ & $172(100.0)$ & Not available \\
Rebound tenderness & $3(18.8)$ & $62(36.0)$ & 0.164 \\
Muscle rigidity & $0(0.0)$ & $8(4.7)$ & 1.00 \\
Leukocytosis & $3(18.8)$ & $99(57.6)$ & 0.003 \\
\hline
\end{tabular}

Values are presented as mean \pm SD or number (\%).

arteries branching from the colonic vasa recta and is drained by a single vein through a narrow pedicle. Because of the limited blood supply and long pedunculated shape with free mobility, the epiploic appendage itself is prone to torsion, resulting in ischemia or hemorrhagic infarction. PEA may be associated with venous thrombosis, and although rare, it could develop without impairing the blood supply $[3,12,14]$.

In adults, the usual clinical symptom of PEA is localized abdominal pain of rapid onset, lasting less than one week. It occurs commonly in the 4th to 5th decades of life, predominantly in men [6, $9,15,16]$. In our study, the mean age of patients with PEA was 40 years, and the incidence in the males was more than 2 times higher than that in females.

The most frequently involved sites of PEA have been reported to be the sigmoid colon and the cecum. Thus, right lower quadrant pain and tenderness are reported in $50-55 \%$ of patients and the left lower quadrant pain in $30 \%[3,13]$. In our study, right and left lower quadrant pain were noted in equal numbers of patients (41.9 $\%)$. Three patients (9.7\%) presented with pain in the right upper quadrant, and two patients (6.5\%) presented with pain in the lower abdomen.

The findings on the physical examination of patients having PEA are non-specific. Usually the abdomen is not distended and soft, showing localized tenderness. The incidence of rebound tenderness is relatively low (approximately 25\%), although it has been reported that rebound tenderness may be noted frequently in patients with PEA $[3,6,7,16]$. Similarly, in the present study, all patients showed localized tenderness, and rebound tenderness was 
noted in 8 patients (25.8\%). None of patients showed abdominal rigidity. In general, nausea, vomiting, anorexia, and other gastrointestinal symptoms are relatively rare, and a fever higher than $38.0^{\circ} \mathrm{C}$ has been reported to be rare although fever may be present $[6,14-$ 16]. In our study, similarly, nausea and vomiting were only noted in 4 patients (12.9\%) and 1 patient (3.2\%), respectively. None of the patients developed a fever, and 2 patients (6.5\%) presented with chills. There is no pathognomonic laboratory test in the diagnosis of PEA. In appendicitis, diverticulitis, and other intra-abdominal inflammatory diseases, leukocytosis is frequently observed; thus, one may also expect leukocytosis to be observed frequently in patient with PEA. However, the white blood cell count has been reported to be normal in most cases of PEA, and in our study, leukocytosis was only observed in 4 patients $(12.9 \%)[6,16,17]$.

The differential diagnoses of PEA are acute appendicitis, diverticulitis, cholecystitis, hemorrhagic ovarian cyst, ovarian torsion, ectopic pregnancy, colorectal cancer, and mesenteric lymphadenitis [1]. In our study, after history taking and physical examination, none of the cases were putatively diagnosed as epiploic appendagitis prior to performing imaging studies such as CT or US. The most common presumptive diagnosis was diverticulitis and acute appendicitis, depending on the location of the pain. Three patients (9.7\%) who presented with right upper quadrant pain were putatively diagnosed as having cholecystitis. Since the clinical characteristics of PEA are nonspecific, it is hardly diagnosed prior to an imaging study. Until the widespread use of CT and US, PEA was a diagnosis of exclusion and, in most cases, was diagnosed incidentally during a laparotomy that was performed for other suspected inflammatory diseases [8]. In one study in which CT was performed on 660 patients who were clinically suspected of having appendicitis or diverticulitis, 11 patients $(2 \%)$ were diagnosed as having PEA [18]. Therefore, as CT increasingly is used for the evaluation of an acute abdomen, the incidence of PEA is likely to increase. In our study, most of PEA was diagnosed after the year 2005.

Epiploic appendagitis can be diagnosed by CT, US, and magnetic resonance imaging $[7,10,19-21]$. The characteristic findings of US are an oval noncompressible hyperechoic mass at the site of maximum tenderness, adjacent to the colon, with no central blood flow depicted on color Doppler US images [7, 10, 19]. However, unless radiologists are experienced and familiar with these conditions, the diagnosis of epiploic appendagitis only by using US may be difficult. CT has been reported to be more reliable than US in defining PEA and is the preferred imaging modality. Thus, CT is required for definite diagnosis in many cases [21]. In our study, US was performed in four patients. Except for 1 patient who was pregnant and on whom CT could not be performed, for the remaining three cases, CT was performed after US for a definite diagnosis. Since the CT findings of PEA were first reported in 1986 by Danielson et al. [9], this condition has become more common in the radiologic differential diagnosis of the acute abdomen.

In normal conditions, epiploic appendages are not detectable on a CT scan unless surrounded by an intraperitoneal fluid such as ascite or hemoperitoneum. Generally, the epiploic appendages have fat attenuation and resemble other adipose structures unless they are inflamed. The characteristic CT findings of PEA are an oval fatty mass less than $5 \mathrm{~cm}$ in diameter adjacent to the colon, with a thickened hyperdense rim that represents a swelling of the serosa with fibrino-leukocytic exudates. In some cases, a dot or fine line of high attenuation may be noted in the fatty oval mass, presumably representing the thrombosed vein of the epiploic appendage. In addition, PEA might present a lobulated appearance due to two or more affected, contiguous, epiploic appendages in close proximity $[7,10,21,22]$. In our study, except for 1 case, a fatty oval mass with a hyperdense rim adjacent to the colon was observed, and in 2 cases (6.5\%), a fine line of high attenuation was observed in the center of the fatty mass. In one case, which was misdiagnosed as a GIST in the small bowel, the mass showed a lobulated appearance adjacent to the descending colon. Such characteristic findings of PEA have been shown to disappear as clinical symptoms are resolved [10]. In our study, follow-up CT was performed in two patients after discharge, and the characteristic findings of PEA were not seen on the follow-up images.

In the past, the standard treatment for PEA was surgical excision because it was diagnosed during laparotomy in most cases $[1,8$, 14]. Rarely, PEA may result in adhesion due to inflammation in adjacent tissues, subsequent ileus, intussusceptions, peritonitis, and abscess formation. For such cases, surgical treatment is necessary $[1,23]$. However, whether recurrence is frequent in patient with PEA if not treated surgically is still a controversial issue. Sand et al. [17] reported that in conservatively treated patients, symptoms recurred in $40 \%$ of the cases; thus, surgical therapy is favored to prevent recurrence, inflammation induced adhesions and other less common complications, and laparoscopic interventions are suggested for the treatment of PEA. For recurred cases, surgical treatments may be necessary. Nonetheless, since conservative management for PEA was first reported in 1968 by Epstein and Lempke [24], PEA has been reported to be a self limiting disease that is resolved spontaneously. Most patients recover with conservative management, such as NSAID, in less than four weeks. Thus, antibiotics or surgical treatments are not routinely required $[6,16,21$, 24]. In our study, all patients recovered within one week after conservative management, and recurrence was not observed during the follow-up period. Most patients were treated with antibiotics, but one was not. This is thought to be due to the fact that PEA itself is not familiar to the clinician, and despite the imaging diagnosis of PEA, experience with its treatment is not sufficient; thus, antibiotics are administered excessively. In addition, surgery was performed on one patient. In that case, a mass in the descending colon showing a lobulated appearance was close to the small bowel and had thus been misdiagnosed as a GIST in the small bowel.

Clinically, PEA manifests with acute onset of pain, often in the left or right lower quadrant, and this symptom often leads to its being misdiagnosed as diverticulitis or acute appendicitis. In our 
study, the presumptive diagnosis after history taking and physical examination was diverticulitis or acute appendicitis in most cases. Particularly, in cases with pain in the left lower quadrant, the symptoms are similar to those of diverticulitis. In general, acute diverticulitis occurs in more elderly patients in comparison to PEA. Acute diverticulitis is more likely to manifest with evenly distributed lower abdominal pain and to be associated with nausea, vomiting, fever, leukocytosis, and rebound tenderness [16, 25]. In contrast, most patients with PEA show localized tenderness in the right or left lower quadrant. Associated gastrointestinal symptoms are rare, and most patients have a normal white blood cell count and body temperature. No association between obesity and PEA has been established $[2,14,16]$. In our study, the mean BMI in patients with PEA was $25.9 \pm 3.5 \mathrm{~kg} / \mathrm{m}^{2}$. When compared with patients with diverticulitis, the mean BMI in patients with PEA was significantly higher. According to the location of the disease, patients with left colonic diverticulitis were older than patients with left PEA (69.7 \pm 13.3 vs. $41.4 \pm 11.9, \mathrm{P}<0.001)$. Fever $(40.0 \%$ vs. $0 \%, \mathrm{P}=0.017)$ and leucocytosis ( $80.0 \%$ vs. $6.7 \%, \mathrm{P}<0.001)$ were more frequently observed in patients with left colonic diverticulitis. In addition, leucocytosis was more frequently observed in patients with right colonic diverticulitis (57.6\% vs. 18.8\%, $\mathrm{P}=0.003)$. Therefore, in patients who are relatively young and without fever or leukocytosis and who present with left lower quadrant pain and localized tenderness, the possibility of PEA rather than diverticulitis should be considered. However, because the number of subjects in our study was relatively small, further study on a larger number of cases is necessary to establish the clinical characteristics of PEA.

Many patients visit emergency rooms for abdominal pain with various causatives. It is important for the surgeon to make a decision whether or not to perform surgery based on history taking and physical examination. Nonetheless, recently, a trend of depending on the results of imaging studies such as CT or US has increased, so in many cases, patients with an acute abdomen are diagnosed by using CT findings. However, for patients visiting hospitals at night, CT findings are reported by on-duty residents of the Department of Radiology rather than radiology specialists with abundant clinical experience, and treatments are determined based on such findings. From our experience, because there are some cases that cannot be diagnosed as epiploic appendagitis even after imaging studies and in which unnecessary treatment is performed, the surgeons who ultimately determine the treatment modality should be familiar with this type of uncommon disease. Thus, although rare, the possibility of epiploic appendagitis should be considered in patients presenting with localized lower quadrant pain and tenderness without associated symptoms and signs. In addition, recognition of this condition on CT findings may allow appropriate management of an acute abdomen and help prevent unnecessary surgery or antibiotic therapy.

Our study was performed to find the clinical characteristics of PEA through analysis and comparison with diverticulitis, which is most commonly differentiated, depending on the location of the pain. Unlike patients with diverticulitis, patients with PEA are relatively young and obese, and show localized tenderness, but no associated gastrointestinal symptoms, such as fever and leukocytosis. However, in general, the two diseases are indistinguishable on the basis of clinical manifestations, and diagnosing PEA based on such clinical characteristics only is difficult. Therefore, although rare, recognition of PEA and knowledge of its radiologic findings and natural course are necessary for its diagnosis and appropriate treatment.

PEA is very rare, and the symptom and signs are non-specific, which often leads to clinical misdiagnosis; thus, PEA is ultimately diagnosed by using radiological findings. Nonetheless, it is important to suspect PEA for relatively young patients with an acute abdomen that show only tenderness without associated general symptoms and signs. In addition, for diagnosis and appropriate treatment, it is necessary to recognize the natural course and radiologic findings of the disease.

\section{CONFLICT OF INTEREST}

No potential conflict of interest relevant to this article was reported.

\section{REFERENCES}

1. Carmichael DH, Organ CH Jr. Epiploic disorders. Conditions of the epiploic appendages. Arch Surg 1985;120:1167-72.

2. Dockerty MB, Lynn TE, Waugh JM. A clinicopathologic study of the epiploic appendages. Surg Gynecol Obstet 1956;103:423-33.

3. Thomas JH, Rosato FE, Patterson LT. Epiploic appendagitis. Surg Gynecol Obstet 1974;138:23-5.

4. Fieber SS, Forman J. Appendices epiploicae: clinical and pathological considerations: report of three cases and statistical analysis on one hundred five cases. AMA Arch Surg 1953;66:329-38.

5. Desai HP, Tripodi J, Gold BM, Burakoff R. Infarction of an epiploic appendage: review of the literature. J Clin Gastroenterol 1993;16: 323-5.

6. Legome EL, Belton AL, Murray RE, Rao PM, Novelline RA. Epiploic appendagitis: the emergency department presentation. J Emerg Med 2002;22:9-13.

7. Rioux M, Langis P. Primary epiploic appendagitis: clinical, US, and CT findings in 14 cases. Radiology 1994;191:523-6.

8. Shehan JJ, Organ C, Sullivan JF. Infarction of the appendices epiploicae. Am J Gastroenterol 1966;46:469-76.

9. Danielson K, Chernin MM, Amberg JR, Goff S, Durham JR. Epiploic appendicitis: CT characteristics. J Comput Assist Tomogr 1986;10:142-3.

10. Molla E, Ripolles T, Martinez MJ, Morote V, Rosello-Sastre E. Primary epiploic appendagitis: US and CT findings. Eur Radiol 1998; 8:435-8.

11. Rao PM, Rhea JT, Wittenberg J, Warshaw AL. Misdiagnosis of primary epiploic appendagitis. Am J Surg 1998;176:81-5.

12. ROSS JA. Vascular loops in the appendices epiploicae; their anat- 
omy and surgical significance, with a review of the surgical pathology of appendices epiploicae. Br J Surg 1950;37:464-6.

13. Vinson DR. Epiploic appendagitis: a new diagnosis for the emergency physician. Two case reports and a review. J Emerg Med 1999; 17:827-32.

14. Ghahremani GG, White EM, Hoff FL, Gore RM, Miller JW, Christ ML. Appendices epiploicae of the colon: radiologic and pathologic features. Radiographics 1992;12:59-77.

15. Legome EL, Sims C, Rao PM. Epiploic appendagitis: adding to the differential of acute abdominal pain. J Emerg Med 1999;17:823-6.

16. Son HJ, Lee SJ, Lee JH, Kim JS, Kim YH, Rhee PL, et al. Clinical diagnosis of primary epiploic appendagitis: differentiation from acute diverticulitis. J Clin Gastroenterol 2002;34:435-8.

17. Sand M, Gelos M, Bechara FG, Sand D, Wiese TH, Steinstraesser L, et al. Epiploic appendagitis: clinical characteristics of an uncommon surgical diagnosis. BMC Surg 2007;7:11.

18. Rao PM, Novelline RA. Case 6: primary epiploic appendagitis. Radiology 1999;210:145-8.

19. Danse EM, Van Beers BE, Baudrez V, Pauls C, Baudrez Y, Kartheus- er A, et al. Epiploic appendagitis: color Doppler sonographic findings. Eur Radiol 2001;11:183-6.

20. Sirvanci M, Balci NC, Karaman K, Duran C, Karakas E. Primary epiploic appendagitis: MRI findings. Magn Reson Imaging 2002; 20:137-9.

21. Hiller N, Berelowitz D, Hadas-Halpern I. Primary epiploic appendagitis: clinical and radiological manifestations. Isr Med Assoc J 2000;2:896-8.

22. Ng KS, Tan AG, Chen KK, Wong SK, Tan HM. CT features of primary epiploic appendagitis. Eur J Radiol 2006;59:284-8.

23. Romaniuk CS, Simpkins KC. Case report: pericolic abscess secondary to torsion of an appendix epiploica. Clin Radiol 1993;47: 216-7.

24. Epstein LI, Lempke RE. Primary idiopathic segmental infarction of the greater omentum: case report and collective review of the literature. Ann Surg 1968;167:437-43.

25. Jang JI, Lim YS, Choi JW, Lee YS. Management of right colon diverticulitis. J Korean Soc Coloproctol 2010;26:22-8. 